\title{
Icariin protects against cage layer osteoporosis by intervening in steroid biosynthesis and glycerophospholipid metabolism
}

\author{
Zhengwang $\mathrm{Yu}^{1,2 \dagger}$, Jie Huang ${ }^{1,2+}$ and Zhongxin Zhou ${ }^{1,2^{*}}$
}

\begin{abstract}
Cage layer osteoporosis (CLO) is a common bone metabolism disease in the breeding industry of China. However, effective prevention for CLO has not been developed. Icariin (ICA), the main bioactive component of the Chinese herb Epimedium, has been shown to have good therapeutic effects on bone-related diseases. In this study, the effects of ICA were further evaluated in a low-calcium diet-induced CLO, and a serum metabolomics assay was performed to understand the underlying mechanisms. A total of 144 31-wk-old Lohmann pink-shell laying hens were randomly allocated to 4 groups with 6 replicates of 6 hens per replicate. The 4 dietary treatment groups consisted of a basal diet (3.5\% calcium), a low-calcium diet (2.0\% calcium), and a low-calcium diet supplemented with 0.5 or $2.0 \mathrm{~g} / \mathrm{kg} \mathrm{ICA}$. The results showed that ICA exerted good osteoprotective effects on low-calcium diet-induced CLO. ICA significantly increased femur bone mineral density, improved bone microstructure, decreased bone metabolic level, and upregulated mRNA expression of bone formation genes in femoral bone tissue. Serum untargeted metabolomics analysis showed that 8 metabolite levels were significantly changed after ICA treatment, including increased contents of 7-dehydrocholesterol, 7oxocholesterol, desmosterol, PC (18:1(9Z)/18:1(9Z)), PS (18:0/18:1(9Z)), N,N-dimethylaniline and 2-hydroxy-butanoic acid and decreased N2,N2-dimethylguanosine. Metabolic pathway analysis based on the above 8 metabolites indicated that ICA mainly perturbed steroid biosynthesis and glycerophospholipid metabolism. These findings suggest that ICA can effectively prevent bone loss in low-calcium diet-induced CLO by mediating steroid biosynthesis and glycerophospholipid metabolism and provide new information for the regulation of bone metabolic diseases.
\end{abstract}

Keywords: Osteoporosis, Laying hen, Bone mineral density, Icariin, Metabolomics

\section{Introduction}

Laying hens have recently been suggested as an interesting animal model for studying osteoporosis (RodriguezNavarro et al. 2018). Cage layer osteoporosis (CLO) is also a common bone metabolic disease in high-yielding laying

\footnotetext{
*Correspondence: zxzhouwhu@163.com; zhongxinzhou@mail.hzau.edu.cn ${ }^{\dagger}$ Zhengwang Yu and Jie Huang contributed equally to this work. 'Key Lab of Agricultural Animal Genetics, Breeding and Reproduction of Ministry of Education, Department of Animal Nutrition and Feed Science, College of Animal Sciences \& Technology, Huazhong Agricultural University, Wuhan, P. R. China ${ }^{2}$ Cooperative Innovation Center for Sustainable Pig Production, Huazhong Agricultural University, Wuhan, P. R. China
}

hens (Whitehead and Fleming 2000). As cage layer is still the main breeding mode in China, CLO has caused huge economic losses and been involved in serious animal welfare issues (Tarlton et al. 2013). However, effective prevention for CLO has not been developed.

CLO is considered to be directly related to the excessive absorption of structural bone, which leads to a reduction in bone mass and strength (Whitehead 2004). Studies have also shown that metabolic imbalance of endogenous calcium (Ca) is an important cause of excessive absorption of structural bone in caged laying hens

(c) The Author(s). 2021 Open Access This article is licensed under a Creative Commons Attribution 4.0 International License, which permits use, sharing, adaptation, distribution and reproduction in any medium or format, as long as you give appropriate credit to the original author(s) and the source, provide a link to the Creative Commons licence, and indicate if changes were made. The images or other third party material in this article are included in the article's Creative Commons licence, unless indicated otherwise in a credit line to the material. If material is not included in the article's Creative Commons licence and your intended use is not permitted by statutory regulation or exceeds the permitted use, you will need to obtain permission directly from the copyright holder. To view a copy of this licence, visit http://creativecommons.org/licenses/by/4.0/ The Creative Commons Public Domain Dedication waiver (http://creativecommons.org/publicdomain/zero/1.0/) applies to the data made available in this article, unless otherwise stated in a credit line to the data. 
(Jiang et al. 2019). Laying hens need a large amount of $\mathrm{Ca}$ for eggshell formation every day. Ca in part is provided by diet and the other part comes from skeleton absorption (Kim et al. 2012). Ca absorbed by laying hens is deposited in medullary bone, which acts as a dynamic $\mathrm{Ca}$ reservoir for eggshell formation. Under constant and high-intensity egg production pressure, laying hens not only mobilize $\mathrm{Ca}$ from medullary bone for eggshell formation but also absorb Ca from structural bone (cortical bone) (Casey-Trott et al. 2017). Once Ca content in the diet is insufficient for a long time, osteoclasts will enhance the absorption of cortical bone, which can lead to a progressive loss of cortical bone and subsequently skeletal fragility as well as great susceptibility to fractures during the laying period (Aguado et al. 2015). Eventually, CLO occurs.

In recent years, medicinal plants have attracted much attention in the prevention and treatment of osteoporosis without causing side effects (Zhao et al. 2018). Epimedium is a traditional Chinese herb that has been proven to be effective in fractures, joint disease and sexual dysfunction in humans (Indran et al. 2016). Icariin (ICA), the main bioactive component isolated from Epimedium ( $\mathrm{Li}$ et al. 2015), has been shown to have a good therapeutic effect on osteoporosis in humans and some animal models, such as ovariectomized, glucocorticoidand diabetic-induced osteoporosis rat models (Nian et al. 2009; Feng et al. 2013; Qi et al. 2019). ICA has been proven to promote bone health by regulating multiple bone metabolism pathways, including osteogenic pathways, e.g., BMP, Wnt and MAPK, and osteoclastic pathways, e.g., OPG/RANKL/RANK (Wei et al. 2016). Bone resorption inhibitors are the most commonly used method to treat osteoporosis, but this method can only relieve bone loss (Khosla and Hofbauer 2017). ICA can not only effectively inhibits bone resorption but also stimulates osteogenic differentiation of bone marrowderived mesenchymal stem cells into osteoblasts and simultaneously stimulates bone formation activity of osteoblasts (Wang et al. 2018).

As a rapidly developing "omics" tool, metabolomics can qualitatively and quantitatively measure small endogenous metabolites in tissues, cells or biological fluids and detect their metabolic changes (Johnson et al. 2016). Metabolomics could quickly screen out differential metabolites and further reveal the related metabolic pathways (Lv et al. 2016). It has been successfully used in osteoporosis research and is a powerful tool for biomarker identification and quantitation (Moayyeri et al. 2018).

Our previous research showed that dietary ICA supplementation was beneficial to bone health in naturally aging older caged laying hens (Huang et al. 2020a). To further confirm the preventive effects of ICA on CLO, the present study established a low $\mathrm{Ca}$ diet-induced osteoporosis model in caged laying hens. The underlying mechanisms were characterized by ultrahighperformance liquid chromatography coupled with quadrupole time-of-flight/mass spectrometry (UHPLCQTOF/MS)-based untargeted metabolomics assays.

\section{Materials and methods \\ Animals and experiment design}

The present study was approved by the Institutional Animal Care and Use Committee of Huazhong Agricultural University with an approval number ( $\mathrm{HZAUCH}-$ 2018-010) and was carried out in accordance with the guidelines. A total of 144 31-week-old Lohmann pinkshell laying hens from a commercial farm in Hubei Province, China, were used in this study. After a 1-week adaptation period, hens were randomly allocated to 4 groups with 6 replicates of 6 hens per replicate for a rearing period of 9 weeks. The control (CON) group was fed a basal diet $(3.5 \% \mathrm{Ca})$, the low $\mathrm{Ca}$ diet $(\mathrm{LCD})$ group was fed a low $\mathrm{Ca}$ diet $(2.0 \% \mathrm{Ca})$, and the ICA1 and ICA2 groups were fed low $\mathrm{Ca}$ diets supplemented with ICA at 0.5 or $2.0 \mathrm{~g} / \mathrm{kg}$ feed. ICA (HPLC>60\%) used in this study was obtained from the Shaanxi Sciphar Natural Products Co., Ltd. (Xi'an, China). The basal diet and low Ca diet formulation and nutrient levels were summarized in Table S1.

\section{Management and sample collections}

Hens were randomly assigned to cages $(80 \mathrm{~cm}$ width $\times 80 \mathrm{~cm}$ length $\times 40 \mathrm{~cm}$ height) with 6 hens per cage. They were kept in an environmentally controlled room with ad libitum feeding and watering with temperature controlled at $22^{\circ} \mathrm{C}$ and $16 \mathrm{~h} / \mathrm{d}$ of illumination throughout the entire experimental period. At the end of the experiment, 1 hen from each replicate was randomly selected, blood samples were individually collected from the wing vein and then centrifuged at $3500 \mathrm{~g}$ at $4{ }^{\circ} \mathrm{C}$ for $15 \mathrm{~min}$ to obtain serum and stored at $-80^{\circ} \mathrm{C}$. In addition, hens were slaughtered to collect femurs and tibias.

\section{Bone mineral density (BMD) measurement}

Dual energy X-ray absorptiometry (DXA, InAlyzer, MEDIKORS Inc., Korea) was employed to measure BMD of right femurs and tibias. Muscle and adipose tissue were removed from bones before testing. Bones were temporarily stored at $4{ }^{\circ} \mathrm{C}$, and sample testing was completed within $24 \mathrm{~h}$ after separation. According to the operator's manual, a standard calibration block was used to calibrate the device before testing. The bone samples were placed in a fixed area ready for scanning with the optimum mode in 3 times per sample in $3 \mathrm{~min}$. Detection sensitivity was $0.001 \mathrm{~g} / \mathrm{cm}^{2}$. 


\section{Bone histomorphometry}

The left tibias were used for bone histomorphometry analysis, including cortical area ratio (\%Ct. Ar), cortical width $(\mathrm{CW})$, trabecular area ratio (\% Tb. Ar), trabecular thickness (Tb. Th), trabecular separation (Tb. Sp), and trabecular number (Tb. N). The preparation and staining methods of bone tissue sections were described by Huang et al. (2020b). First, total tissue area (T. Ar) of cancellous bone, trabecular bone area (Tb. Ar), trabecular perimeter (Tb. Pm), cortical area (Ct. Ar) and CW were analyzed in the slice field of view using Image-Pro Plus V. 6.0. The calculation methods of each analysis index are as follows:

$$
\begin{aligned}
& \text { \%Ct.Ar }=\text { Ct.Ar } / \mathrm{T} . A r \times 100 . \\
& \% \mathrm{~Tb} . A r=\mathrm{Tb} . A r / \mathrm{T} . \mathrm{Ar} \times 100 . \\
& \text { Tb.Th }=(2000 / 1.199) \times(\mathrm{Tb} . \mathrm{Ar} / \mathrm{Tb} . \mathrm{Pm}) \\
& \text { Tb.Sp }=(2000 / 1.19) \times(\mathrm{T} . \mathrm{Ar}-\mathrm{Tb} . \mathrm{Ar}) / \mathrm{Tb} . \mathrm{Pm} . \\
& \text { Tb.N }=(1.199 / 2) \times(\mathrm{Tb} . \mathrm{Pm} / \mathrm{Tb} . \mathrm{Ar}) .
\end{aligned}
$$

\section{Serum bone metabolism index}

Activities of alkaline phosphatase (ALP) and tartrateresistant acid phosphatase (TRACP) and levels of $\mathrm{Ca}$ in serum were measured by specific assay kits (Nanjing Jiancheng Bioengineering Institute, Nanjing, China). Concentration of osteocalcin $(\mathrm{OCN})$ in serum was measured using ELISA kits (CH50029, Bioswamp Life Science Lab., Wuhan, China) according to the manufacturer's instructions.

\section{Serum redox state}

Activities of total antioxidant capacity (T-AOC), total superoxide dismutase (T-SOD), glutathione peroxidase (GSH-Px) and content of malondialdehyde (MDA) in serum were determined using analysis kits (Nanjing Jiancheng Bioengineering Institute, Nanjing, China) according to the manufacturer's instructions.

\section{Total RNA extraction and real-time quantitative PCR}

Part of left femur tissues were completely ground, and TRIzol Reagent (Invitrogen, USA) was added to extract total RNA according to the manufacturer's protocol. Reverse transcription reaction was performed using ABScript II RT Master Mix (ABclonal Technology, Wuhan, China). Complementary DNA (cDNA) samples were appropriately diluted for primer testing and subsequent real-time quantitative PCR. The forward and reverse primer sequences of runt-related transcription factor 2 (RUNX2), osteoprotegerin (OPG), and receptor activator of nuclear factor kappa-B ligand (RANKL) were designed based on available sequences in NCBI GenBank by using Primer Premier 6.0. All primers were tested on pooled cDNA and summarized in Table S1. $\beta$-actin was chosen as an internal standard to control for normalization purposes. Real-time quantitative PCR was performed using the iTaq Universal SYBR Green Supermix protocol (Bio-Rad, Richmond, CA, USA). Expression in triplicates was assayed, and the results were used for plotting and statistical analysis by $2^{-\Delta \Delta C T}$ method.

\section{Differential metabolites screening}

Serum samples $(100 \mu \mathrm{L}$ per sample) of CON, LCD and ICA2 groups were subjected to metabolite extraction. To validate system stability, a quality control (QC) sample was obtained by mixing $10 \mu \mathrm{L}$ of every prepared serum sample. Metabolite extraction and UHPLCQTOF/MS data processing were described by Huang et al. (2020a). Metabolites with a variable importance projection (VIP) $>1.0$ and $P<0.05$ were selected as differential metabolites.

\section{Correlation and metabolic pathway analysis}

Spearman correlation analysis was employed to investigate the correlation between different metabolites and BMD or bone metabolism index. Furthermore, differential metabolites were imported into MetaboAnalyst V. 4.0, an online data handling system (https://www. metaboanalyst.ca/) to discover relevant metabolic pathways closely related to ICA treatment.

\section{Statistical analysis}

All quantitative data were expressed as the mean \pm standard deviation (SD) and were analyzed using SPSS V. 22.0 (SPSS Inc., Chicago, IL, USA). One-way ANOVA was used for the analysis of group differences. WelchANOVA was employed to measure heterogeneous variances. Post hoc analyses were performed by means of Bonferroni test or Games-Howell post hoc test (following Welch-ANOVA) to characterize the significant effects. A value of $P<0.05$ was considered statistically significant.

\section{Results}

\section{Effects of ICA on BMD of femur and tibia}

Traditionally, BMD is the gold standard for clinical diagnosis of osteoporosis (Eastell et al. 2016). BMD measurement can characterize osteopromotive effects. The LCD group had significantly lower BMD of femur $(P=0.001)$ and tibia $(P=0.014)$ than the CON group (Fig. 1). Supplying ICA at $0.5 \mathrm{~g} / \mathrm{kg}$ feed did not affect femur and tibia BMD, while ICA at $2.0 \mathrm{~g} / \mathrm{kg}$ feed significantly increased femur BMD $(P=0.041)$ and had a tendency to increase tibia BMD $(P=0.079)$ compared with the LCD group. 

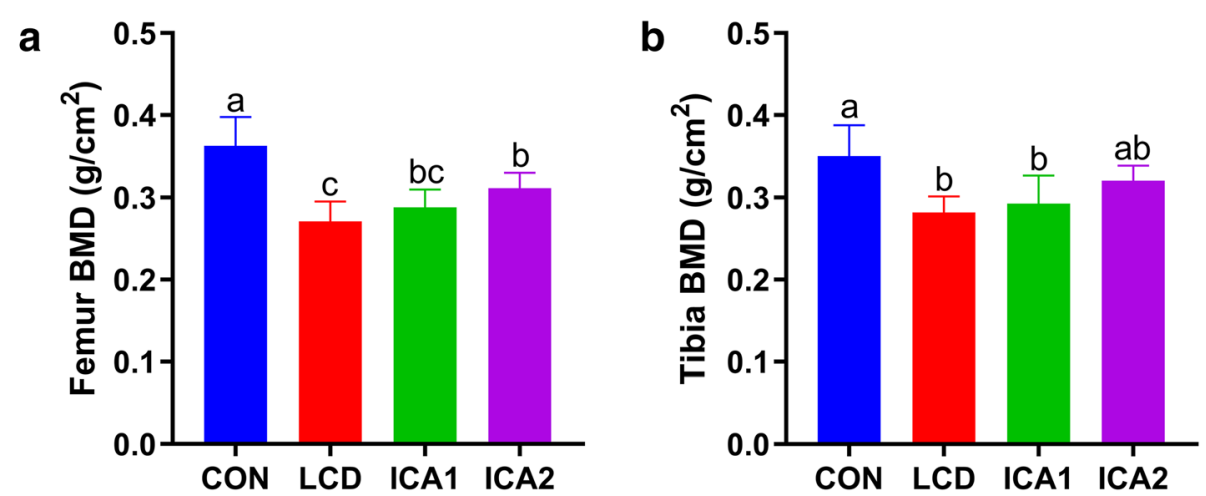

Fig. 1 Effects of ICA supplementation on bone mineral density (BMD) of femur and tibia. a Femur BMD and $\mathbf{b}$ tibia BMD. ICA, icariin. CON, basal diet. LCD, low calcium diet. ICA1, low calcium diet supplied with $0.5 \mathrm{~g} / \mathrm{kg}$ ICA. ICA2, low calcium diet supplied with $2.0 \mathrm{~g} / \mathrm{kg}$ ICA. Data are presented as the mean \pm SD $(n=6)$. Means in each column with different alphabetic letters are significantly $(P<0.05)$ different

\section{Effects of ICA on microstructure of tibia}

Bone histomorphometry of caged laying hens was further analyzed. This tests can intuitively reveal changes in bone microstructure, including a clear distinction between cortical bone and trabecular bone, which is particularly valuable in determining the mechanism of drugs (Recker et al. 2018).

In the LCD group, many large absorption cavities were observed on cortical bone with a reduced area
(Fig. 2a). Trabecular bone also became loose or broken with decreased area. For the ICA treatment groups, absorption cavities were reduced, and trabecular bone structures were more complete than those of the LCD group. In addition, static parameters of bone histomorphometry were analyzed (Fig. $2 \mathrm{~b}$ and $\mathrm{c})$. Compared to the CON group, the LCD group had significantly lower Ct. Ar $(P=0.011)$, Tb. $\operatorname{Ar}(P=0.008)$ and Tb. Th $(P=0.005)$, and higher Tb.

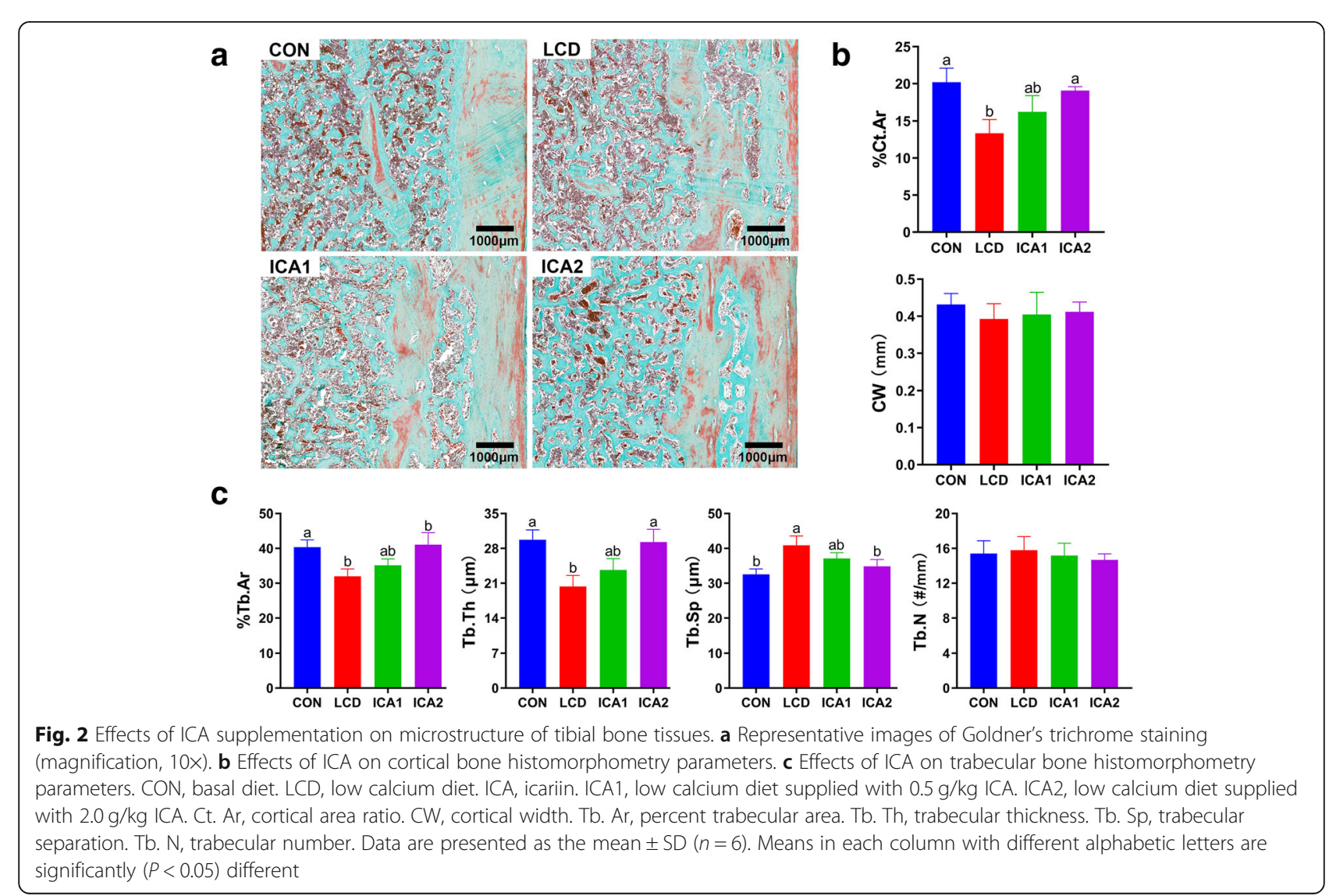


Sp $(P=0.009)$. Supplementation of ICA at $2.0 \mathrm{~g} / \mathrm{kg}$ feed enhanced Ct. Ar $(P=0.022)$, Tb. Ar $(P=0.012)$ and Tb. Th $(P=0.008)$, with lower Tb. Sp $(P=0.038)$ than that of LCD hens. Tb. N and CW did not differ among groups in the study.

\section{Effects of ICA on serum bone metabolism index}

Serum bone turnover markers, including bone formation markers such as ALP and OCN and bone resorption markers such as TRACP, are useful for diagnosis and therapeutic monitoring of bone metabolic disorders (Morris et al. 2017). As shown in Fig. 3, the LCD group had higher activities of ALP $(P<0.001)$ and TRACP $(P=0.006)$ and OCN contents $(P<0.001)$ than the CON group. Supplementation with ICA at $0.5 \mathrm{~g} / \mathrm{kg}$ feed decreased the content of $\mathrm{OCN}(P=0.039)$ compared with that in the LCD group. Supplementation of ICA at $2.0 \mathrm{~g} / \mathrm{kg}$ feed significantly reduced ALP $(P=0.001)$ and TRACP activities $(P<0.001)$ and $\mathrm{OCN}$ content $(P=0.012)$. Serum Ca levels did not differ among treatments in this study (Fig. 3d).

\section{Effects of ICA on serum redox state}

Accumulating studies have shown that oxidative stress is related to the pathophysiology of osteoporosis (Geng et al. 2019; Saelao et al. 2019). Figure 4 showed that serum redox index of the LCD group was significantly affected, with increased MDA levels $(P<0.001)$ and reduced TAOC $(P<0.001)$, T-SOD $(P<0.001)$ and GSH-Px $(P=$ $0.006)$ activities compared to those of CON group. Supplying ICA at $0.5 \mathrm{~g} / \mathrm{kg}$ feed increased T-AOC $(P=0.019)$ and GSH-Px $(P=0.011)$ activities but did not affect MDA and T-SOD activities compared with the LCD group. Furthermore, supplementation with ICA at $2.0 \mathrm{~g} / \mathrm{kg}$ feed significantly decreased MDA levels $\quad(P<0.001)$ and enhanced T-AOC $(P=0.013)$, T-SOD $(P=0.017)$ and GSH-Px $(P<0.001)$ activities compared to the LCD group.

\section{Effects of ICA on relative mRNA expression of bone} metabolism-related genes

RUNX2 is a multifunctional transcription factor that controls skeletal development by regulating the differentiation of mesenchymal stem cells into osteoblasts. RANKL is an essential mediator of osteoclast formation and function,
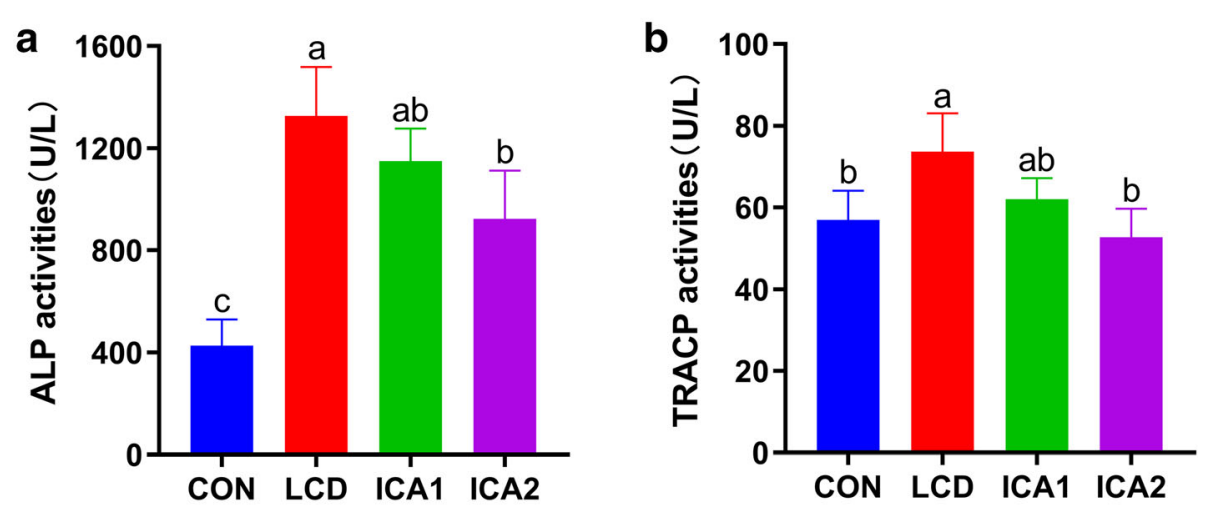

C

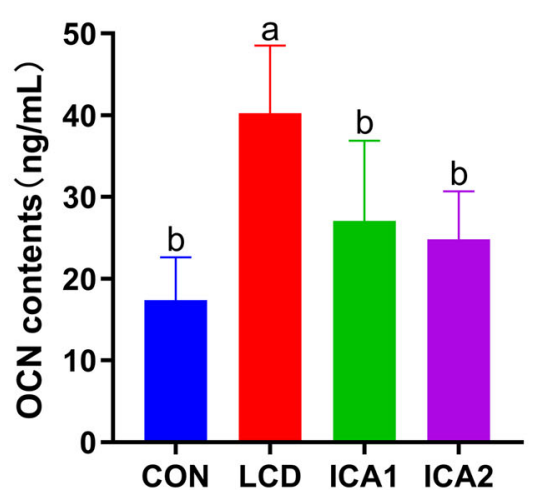

d

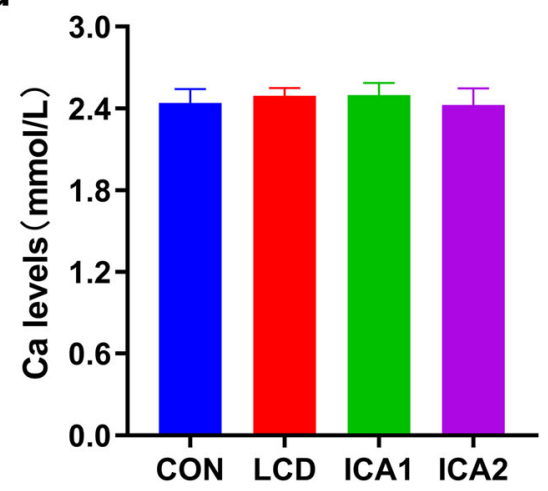

Fig. 3 Effects of ICA supplementation on serum bone metabolism index. a Activities of ALP. $\mathbf{b}$ Contents of OCN. c Activities of TRACP. $\mathbf{d}$ Levels of serum Ca. ALP, alkaline phosphatase. OCN, osteocalcin. TRACP, tartrate resistant acid phosphatase. ICA, icariin. CON, basal diet. LCD, low calcium diet. ICA1, low calcium diet supplied with $0.5 \mathrm{~g} / \mathrm{kg}$ ICA. ICA2, low calcium diet supplied with $2.0 \mathrm{~g} / \mathrm{kg}$ ICA. Data are presented as the mean \pm SD $(n=6)$. Means in each column with different alphabetic letters are significantly $(P<0.05)$ different 
a

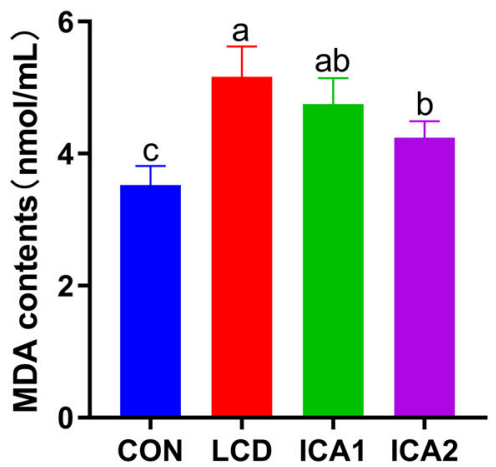

C

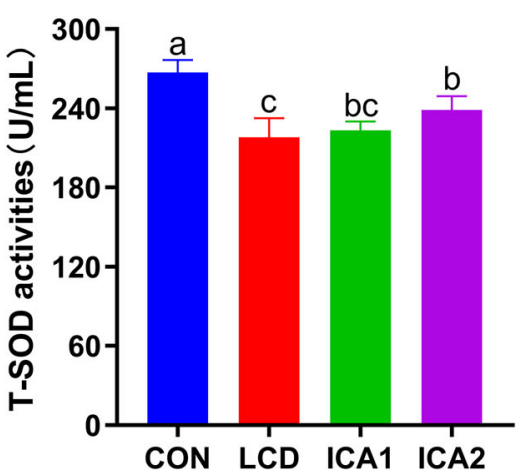

b

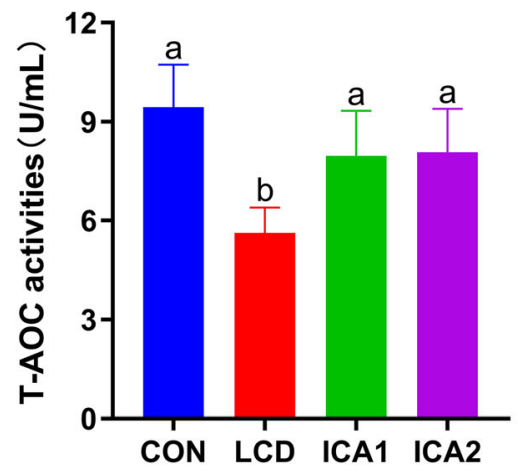

d

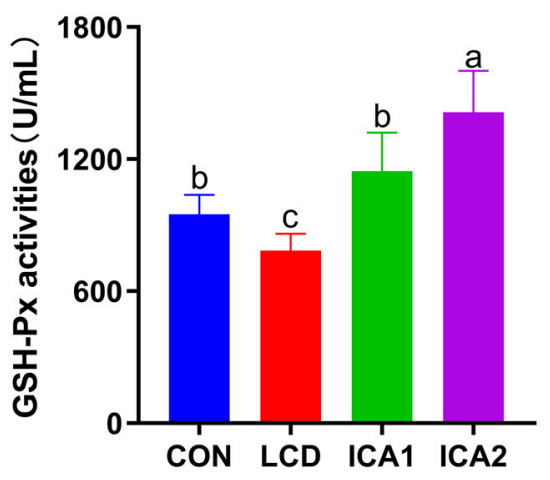

Fig. 4 Effects of ICA supplement on serum redox state. a Contents of MDA. b Activities of T-AOC. c Activities of T-SOD. $\mathbf{d}$ Activities of GSH-PX. MDA, malondialdehyde. T-AOC, total antioxidant capacity. T-SOD, total superoxide dismutase. GSH-Px, glutathione peroxidase. ICA, icariin. CON, basal diet. LCD, low calcium diet. ICA1, low calcium diet supplied with $0.5 \mathrm{~g} / \mathrm{kg}$ ICA. ICA2, low calcium diet supplied with $2.0 \mathrm{~g} / \mathrm{kg}$ ICA. Data are presented as the mean \pm SD $(n=6)$. Means in each column with different alphabetic letters are significantly $(P<0.05)$ different

which exerts bone resorption by binding to receptor activator of nuclear factor kappa-B (RANK) (Martin and Sims 2015). $O P G$ is a soluble receptor and a natural inhibitor of $R A N K L$ which protects bone from excessive resorption by binding to RANKL (Boyce and Xing 2008).

As showed in Fig. 5, LCD significantly decreased expression of RUNX2 ( $P=0.002)$, OPG $(P=0.006)$ and OPG/RANKL $(P<0.001)$ and increased RANKL $(P<0.001)$ compared with the CON group. Supplementation with ICA at $0.5 \mathrm{~g} / \mathrm{kg}$ feed enhanced $R U N X 2(P=0.002)$ and $O P G(P=$ $0.035)$ and decreased $R A N K L(P=0.048)$ levels. Compared with the LCD group, the ICA2 group had higher RUNX2 $(P<0.001)$, OPG $(P<0.001)$ and OPG/RANKL $(P<0.001)$ expression with lower $R A N K L(P<0.001)$ expression.

\section{Quality control and multivariate statistical analysis of} serum metabolomics assay

Under the optimal UHPLC-QTOF/MS conditions, serum samples of CON, LCD and ICA2 groups were

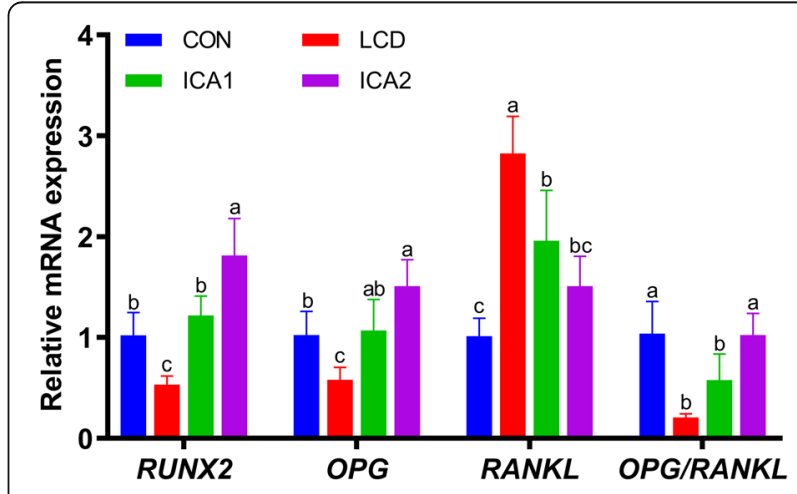

Fig. $\mathbf{5}$ Effects of ICA supplementation on relative mRNA expression of bone metabolism-related genes. RUNX2, runt related transcription factor 2. $O P G$, osteoprotegerin. RANKL, receptor activator of nuclear factor kappa-B ligand. ICA, icariin. CON, basal diet. LCD, low calcium diet. ICA1, low calcium diet supplied with $0.5 \mathrm{~g} / \mathrm{kg}$ ICA. ICA2, low calcium diet supplied with $2.0 \mathrm{~g} / \mathrm{kg}$ ICA. Data are presented as the mean \pm SD $(n=6)$. Means in each column with different alphabetic letters are significantly $(P<0.05)$ different 
analyzed. The obtained metabolic profiles were plotted using principal component analysis (PCA) (Fig. 6a and $\mathrm{b}$ ). PCA revealed clustering trends in metabolomics data, and the plot of PCA scores divided serum samples of 3 groups into chunks, where each point represents an individual sample. A high degree of aggregation in all QC samples was observed in the PCA score plot, indicating good stability of analytical platforms throughout the entire experiment. More sophisticated orthogonal projections to latent structuresdiscriminate analysis (OPLS-DA) was performed to detect whether differential metabolites were responsible for the separation among groups. Figure $6 \mathrm{c}$ and $\mathrm{d}$ showed that these 3 groups were distinguished clearly and the ICA2 group was in the middle of the CON group and LCD group, indicated that ICA played a certain regulatory effect.

Differential metabolites induced by LCD or ICA treatment By defining variable importance projection (VIP) values obtained from OPLS-DA data greater than 1.5 and $P$-values less than 0.05 in Student's t-test analysis
(Fig. 7a), a total of 28 metabolites were screened out as differential metabolites to indicate separation between the CON and LCD groups (Table S1). Similarly, 45 metabolites were screened out as differential metabolites to indicate separation between the ICA2 and LCD groups (Table S1). As showed in Fig. 7b and summarized in Table 1 , there were 8 shared differential metabolites among the 3 groups. A heat map was constructed to visualize changes in those 8 differential metabolites in different groups (Fig. 7c). Among them, 7 metabolite levels (7-dehydrocholesterol, 7-oxocholesterol, desmosterol, PC (18:1(9Z)/18:1(9Z)), PS (18:0/18:1(9Z)), $\mathrm{N}, \mathrm{N}$-dimethylaniline and 2-hydroxy-butanoic acid) in laying hens fed a low calcium diet were significantly decreased, and 1 metabolite (N2,N2-dimethylguanosine) level was increased. After ICA treatment, the 8 metabolite levels were significantly reversed.

\section{Correlation between differential metabolites and BMD or bone metabolism index}

Spearman correlation analysis was employed to investigate correlations between the above 8 different

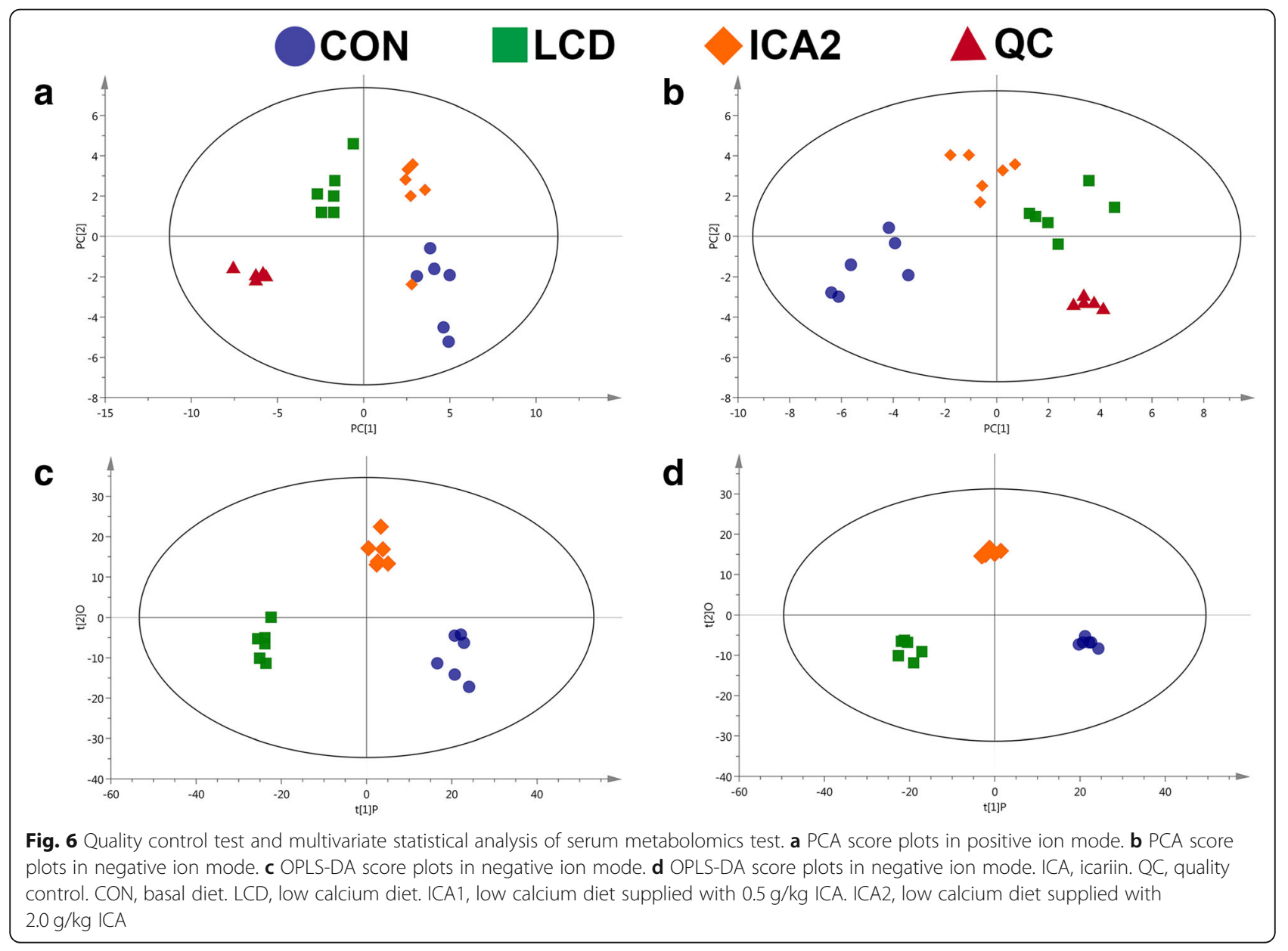


a
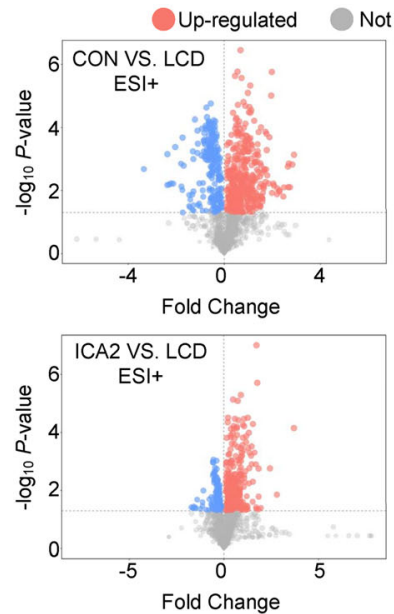

C

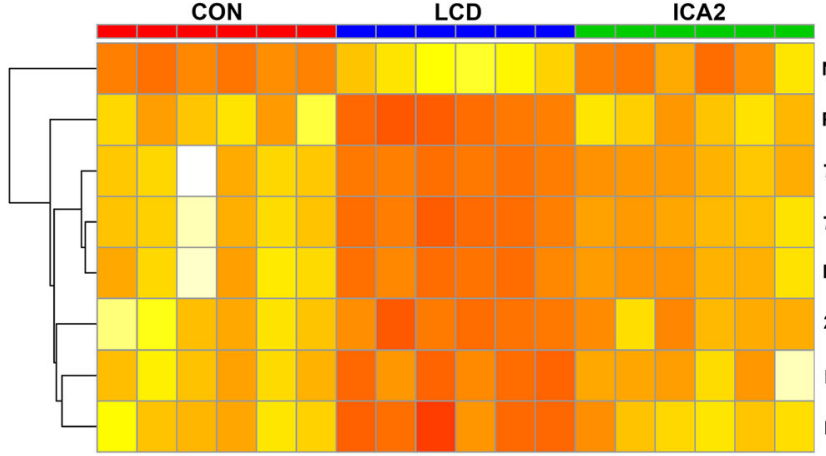

b
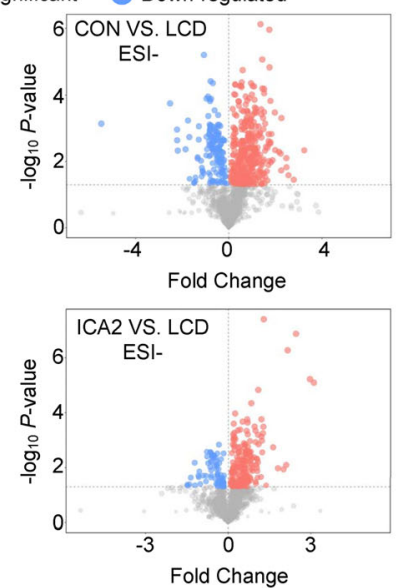

ICA2

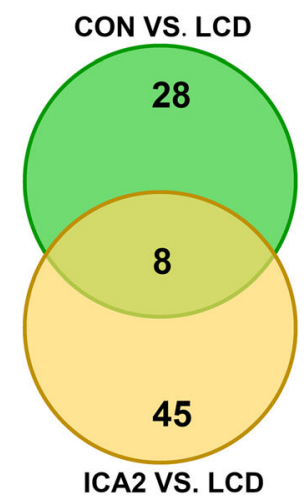

\begin{tabular}{ll} 
N2,N2-Dimethylguanosine & 3 \\
PS (18:0/18:1(9Z)) & 2 \\
7-Oxocholesterol & 1 \\
7-Dehydrocholesterol & 0 \\
Desmosterol & -1 \\
\hline
\end{tabular}

2-Hydroxy-butanoic acid

$\mathrm{N}, \mathrm{N}$-Dimethylaniline

PC (18:1(9Z)/18:1(9Z))

Fig. 7 Differential metabolites induced by ICA treatment. a Volcano map of metabolites for groups (CON vs. LCD and ICA2 vs. LCD) in positive and negative iron modes. $\mathbf{b}$ The shared and unique numbers of differential metabolites in the Venn diagram from CON vs. LCD and ICA2 vs. LCD. c Heat map and hierarchical clustering of 8 shared differential metabolites among the CON, LCD and ICA2 groups. ICA, icariin. CON, base diet. LCD, low calcium diet. ICA2, low calcium diet supplied with $2.0 \mathrm{~g} / \mathrm{kg}$ ICA

metabolites and BMD or bone metabolism index. The results showed that these differential metabolites had a lower correlation with femur and tibia BMD but a higher correlation with all 3 serum bone metabolism indices (Fig. 8). ALP, TRACP and OCN were highly positively correlated with N2,N2-dimethylguanosine while highly negatively correlated with 7dehydrocholesterol, 7-oxocholesterol, desmosterol, PC (18:1(9Z)/18:1(9Z)), PS (18:0/18:1(9Z)), N,N-dimethylaniline and 2-hydroxy-butanoic acid.

Table 1 ICA-induced 8 shared differential metabolites

\begin{tabular}{|c|c|c|c|c|c|c|c|c|c|}
\hline \multirow[t]{2}{*}{ No. } & \multirow[t]{2}{*}{ Metabolite } & \multirow[t]{2}{*}{ Rt (s) } & \multirow[t]{2}{*}{$\mathrm{m} / \mathrm{z}$} & \multicolumn{3}{|c|}{ CON vs. LCD } & \multicolumn{3}{|c|}{ ICA2 vs. LCD } \\
\hline & & & & VIP & Fold change & $P$-value & VIP & Fold change & $P$-value \\
\hline 1 & 7-Dehydrocholesterol & 36.177 & 385.3441977 & 1.59 & 2.94 & 0.003 & 1.88 & 2.26 & $<0.001$ \\
\hline 2 & 7-Oxocholesterol & 34.835 & 401.339414 & 1.61 & 6.74 & 0.008 & 1.86 & 3.59 & 0.002 \\
\hline 3 & Desmosterol & 36.73 & 367.3338581 & 1.54 & 3.11 & 0.008 & 1.75 & 2.09 & 0.003 \\
\hline 4 & PC (18:1(9Z)/18:1(9Z)) & 125.466 & 824.5521835 & 1.52 & 1.51 & $<0.001$ & 1.84 & 1.48 & $<0.001$ \\
\hline 5 & PS (18:0/18:1(9Z)) & 132.1485 & 788.5423368 & 1.62 & 1.84 & 0.002 & 2.15 & 1.78 & $<0.001$ \\
\hline 6 & $\mathrm{~N}, \mathrm{~N}$-Dimethylaniline & 36.52 & 281.1373045 & 1.53 & 1.86 & $<0.001$ & 1.65 & 1.87 & 0.030 \\
\hline 7 & 2-hydroxy-butanoic acid & 179.823 & 103.0396129 & 1.63 & 2.01 & 0.002 & 1.78 & 1.51 & 0.005 \\
\hline 8 & N2,N2-Dimethylguanosine & 180.198 & 310.1146027 & 1.76 & 0.48 & $<0.001$ & 1.79 & 0.58 & 0.003 \\
\hline
\end{tabular}

Notes: Only metabolites with a variable importance projection (VIP) value greater than 1.5 and a $P$-value less than 0.05 were selected as differential metabolites. ICA, icariin. CON, basal diet. LCD, low calcium diet. ICA2, low calcium diet supplied with $2.0 \mathrm{~g} / \mathrm{kg}$ ICA 


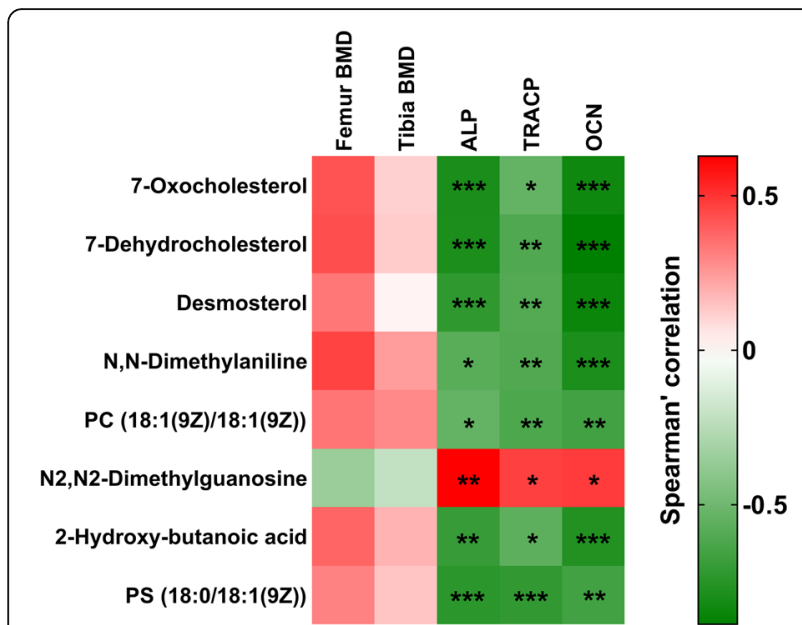

Fig. 8 Correlation between differential metabolites and BMD or bone metabolism index. Different colors (green to red) represent correlation coefficients. ${ }^{*} P<0.05,{ }^{* *} P<0.01,{ }^{* * *} P<0.001$. BMD, bone mineral density. ALP, alkaline phosphatase. OCN, osteocalcin. TRACP, tartrate resistant acid phosphatase

Osteoprotective mechanisms of ICA on LCD-induced CLO To further gain insights into what mechanisms contributed to the osteoprotective effects of ICA on LCDinduced CLO, the above differential metabolites were imported into MetaboAnalyst V. 4.0 to analyze relevant metabolic pathways. A total of 6 metabolic pathways were involved in metabolic alterations related to ICA treatment, including glycerophospholipid metabolism, steroid biosynthesis, linoleic acid metabolism, alphalinolenic acid metabolism, propanoate metabolism and arachidonic acid metabolism (Fig. 9). Among these metabolic pathways, glycerophospholipid metabolism and steroid biosynthesis with impact values of 0.12 and 0.09 were deemed the primary changed pathways related to ICA treatment.

\section{Discussion}

In this study, LCD resulted in a significant decrease in BMD of both femur and tibia in caged laying hens, indicating that CLO was successfully induced. Meanwhile, supplying ICA at $2.0 \mathrm{~g} / \mathrm{kg}$ feed significantly restored femur BMD of caged laying hens fed a low $\mathrm{Ca}$ diet. However, BMD measurement cannot distinguish between cortical bone and trabecular bone and describe changes in bone microstructure (Lorentzon and Cummings 2015). Therefore, bone histomorphometry of caged laying hens was further analyzed. Bone histomorphometry results showed that microstructure of bone tissue was improved with ICA treatments, which was consistent with previous results in a rat/mouse osteoporosis model (Xu et al. 2016; Hu et al. 2017).

Osteoporosis can be divided into 2 types according to the characteristics of bone turnover, high turnover

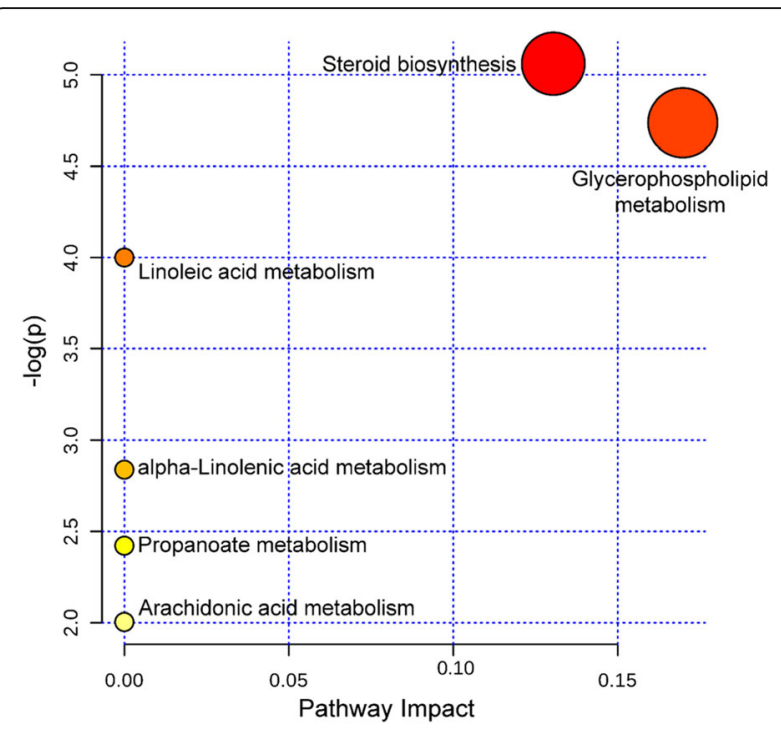

Fig. 9 ICA-induced metabolic pathway perturbations by MetaboAnalyst V. 4.0. Each circle dot represents one metabolic pathway. Circle size and circle color were based on the pathway impact value and $P$-value, respectively

osteoporosis and low turnover osteoporosis, and their respective representatives are postmenopausal osteoporosis and senile osteoporosis (Greenblatt et al. 2017). For serum bone turnover markers in this study, bone formation markers such as serum ALP and OCN and bone resorption markers such as TRACP were significantly increased in the LCD group compared to the basal diet group, indicating a higher bone turnover rate. After ICA supplementation, ALP, OCN and TRACP levels were significantly decreased, suggesting that ICA can more notably affect osteoclast-mediated bone resorption than osteoblast-mediated bone formation in caged laying hens, thereby preventing bone loss.

Serum redox index measurements indicated that laying hens fed a low Ca diet had more oxidative stress. Excessive oxidative stress affected the expression of bone metabolism-related genes and caused an imbalance in bone remodeling in favor of osteoclastogenesis (Domazetovic et al. 2017). Oxidative stress promoted the expression of $R A N K L$, leading to enhanced osteoclastogenesis and bone resorption. Oxidative stress can also inhibit osteoblastic differentiation of bone cells (Bai et al. 2004; Komori 2010). The results from this study showed that ICA reduced oxidative stress and regulated RUNX2, OPG and RANKL mRNA expression to improve the balance of bone resorption and bone formation in favor of osteoblastogenesis for LCD induced CLO.

Metabolic pathway analysis of ICA inducing 8 different metabolites, including 7-dehydrocholesterol, 7-oxocholesterol, desmosterol, PC (18:1(9Z)/18:1(9Z)), PS (18:0/18:1(9Z)), N,N-dimethylaniline, 2-hydroxy- 
butanoic acid and N2,N2-dimethylguanosine, revealed that they were mainly involved in steroid biosynthesis and glycerophospholipid metabolism. It has been reported that epimedium can improve intestinal $\mathrm{Ca}$ absorption in osteoporotic rats, which is positively related to vitamin $D_{3}$ levels (Zhang et al. 2006). Furthermore, 7-dehydrocholesterol is a direct precursor of vitamin $\mathrm{D}_{3}$ (Patwardhan et al. 2015). Therefore, it is reasonable that in caged laying hens, ICA supplementation significantly increased 7-dehydrocholesterol levels, which elevated the concentration of vitamin $D_{3}$ to improve $\mathrm{Ca}$ absorption and bone formation. 7Oxocholesterol is a major oxidation product of cholesterol (oxysterol) (Griffiths et al. 2019). Desmosterol is an intermediate in cholesterol synthesis. Glycerophospholipids, such as PC (18:1(9Z)/18:1(9Z)) and PS (18:0/18:1(9Z)), are the main components of biological membranes (Huang and Freter 2015). In aging bone marrow mesenchymal stromal cells, membrane glycerophospholipid composition and functionality are altered (Kilpinen et al. 2013). Many metabolomics studies found that glycerophospholipid metabolism was involved in metabolic disorders of osteoporosis (Luo et al. 2018; Xu et al. 2018; Zhang et al. 2018).

In summary, this study demonstrated that ICA had great potential to prevent LCD-induced CLO. An untargeted metabolomics strategy indicated that ICA might exert osteoprotective effects by intervening in steroid biosynthesis and glycerophospholipid metabolism.

\section{Supplementary Information}

The online version contains supplementary material available at https://doi. org/10.1186/s44149-021-00001-z.

Additional file 1: Supplementary Tables S1-S4. Table S1 Basal diet and low calcium diet formulation and nutrient levels. Table S2 Primers used for the quantitative polymerase chain reaction. Table S3 Differential metabolites identified by UHPLC-MS/MS between CON and LCD groups. Table S4 Differential metabolites identified by UHPLC-MS/MS between ICA2 and LCD groups.

\section{Acknowledgments}

This work was supported by the National Key Research and Development Program of China (No. 2017YFD0502200, No. 2016YFD0501210) and the Fundamental Research Funds for the Central Universities (No. 2662014BQ023).

\section{Authors' contributions}

All authors have read and approved the final version of the manuscript.

\section{Declarations}

\section{Competing interests}

The authors declare no conflicts of interest.
Received: 10 September 2020 Accepted: 25 December 2020

Published online: 23 April 2021

\section{References}

Aguado, E., F. Pascaretti-Grizon, E. Goyenvalle, M. Audran, and D. Chappard. 2015. Bone mass and bone quality are altered by hypoactivity in the chicken. PLoS One 10 (1): e116763. https://doi.org/10.1371/journal.pone.0116763.

Bai, X., D. Lu, J. Bai, H. Zheng, Z. Ke, X. Li, and S. Luo. 2004. Oxidative stress inhibits osteoblastic differentiation of bone cells by ERK and NF-KB. Biochemical and Biophysical Research 314 (1): 197-207. https://doi.org/10.101 6/j.bbrc.2003.12.073.

Boyce, B.F., and L. Xing. 2008. Functions of RANKL/RANK/OPG in bone modeling and remodeling. Archives of Biochemistry and Biophysics 473 (2): 139-146. https://doi.org/10.1016/j.abb.2008.03.018.

Casey-Trott, T.M., D.R. Korver, M.T. Guerin, V. Sandilands, S. Torrey, and T.M. Widowski. 2017. Opportunities for exercise during pullet rearing, part ii: Long-term effects on bone characteristics of adult laying hens at the end-oflay. Poultry Science 96 (8): 2518-2527. https://doi.org/10.3382/ps/pex060.

Domazetovic, V., G. Marcucci, T. lantomasi, M.L. Brandi, and M.T. Vincenzini. 2017. Oxidative stress in bone remodeling: role of antioxidants. Clinical Cases in Mineral and Bone Metabolism 14 (2): 209-216. https://doi.org/10.11138/ $\mathrm{ccmbm} / 2017.14 .1 .209$.

Eastell, R., T.W. O'Neill, L.C. Hofbauer, B. Langdahl, I.R. Reid, D.T. Gold, and S.R. Cummings. 2016. Postmenopausal osteoporosis. Nature Reviews. Disease Primers 2 (1): 16069. https://doi.org/10.1038/nrdp.2016.69.

Feng, R., L. Feng, Z. Yuan, D. Wang, F. Wang, B. Tan, S. Han, T. Li, D. Li, and Y. Han 2013. Icariin protects against glucocorticoid-induced osteoporosis in vitro and prevents glucocorticoid-induced osteocyte apoptosis in vivo. Cell Biochemistry and Biophysics 67 (1): 189-197. https://doi.org/10.1007/s12013013-9533-8.

Geng, Q., H. Gao, R. Yang, K. Guo, and D. Miao. 2019. Pyrroloquinoline quinone prevents estrogen deficiency-induced osteoporosis by inhibiting oxidative stress and osteocyte senescence. International Biological Sciences 15 (1): 5868. https://doi.org/10.7150/ijbs.25783.

Greenblatt, M.B. J.N. Tsai, and M.N. Wein. 2017. Bone turnover markers in the diagnosis and monitoring of metabolic bone disease. Clinical Chemistry 63 (2): 464-474. https://doi.org/10.1373/clinchem.2016.259085.

Griffiths, WJ., E. Yutuc, J. Abdel-Khalik, P.J. Crick, T. Hearn, A. Dickson, B.W. Bigger, T.H. Wu, A. Goenka, A. Ghosh, et al. 2019. Metabolism of non-enzymatically derived oxysterols: clues from sterol metabolic disorders. Free Radical Biology and Medicine 144: 124-133. https://doi.org/10.1016/j.freeradbiomed.2019.04.020.

Hu, J., Z. Mao, S. He, Y. Zhan, R. Ning, W. Liu, B. Yan, and J. Yang. 2017. Icariin protects against glucocorticoid induced osteoporosis, increases the expression of the bone enhancer DEC1 and modulates the PI3K/Akt/GSK3ß/ $\beta$-catenin integrated signaling pathway. Biochemical Pharmacology 136: 109121. https://doi.org/10.1016/j.bcp.2017.04.010.

Huang, C., and C. Freter. 2015. Lipid metabolism, apoptosis and cancer therapy. International Journal of Molecular Sciences 16 (1): 924-949. https://doi.org/1 0.3390/ijms16010924.

Huang, J., Y. Hu, X. Tong, L. Zhang, Z. Yu, and Z. Zhou. 2020a. Untargeted metabolomics revealed therapeutic mechanisms of icariin on low bone mineral density in older caged laying hens. Food \& Function 11 (4): 3201 3212. https://doi.org/10.1039/C9FO02882J.

Huang, J., X.F. Tong, Z.W. Yu, Y.P. Hu, L. Zhang, Y. Liu, and Z.X. Zhou. 2020b. Dietary supplementation of total flavonoids from Rhizoma Drynariae improves bone health in older caged laying hens. Poultry Science 99 (10): 5047-5054. https://doi.org/10.1016/j.psj.2020.06.057.

Indran, I.R., R.L.Z. Liang, T.E. Min, and E. Yong. 2016. Preclinical studies and clinical evaluation of compounds from the genus Epimedium for osteoporosis and bone health. Pharmacology and Therapeutics 162: 188-205. https://doi.org/1 0.1016/.jpharmthera.2016.01.015

Jiang, S., X.L. Wu, M.L. Jin, X.Z. Wang, Q. Tang, Y.X. Sun, and H.W. Cheng. 2019. Pathophysiological characteristics and gene transcriptional profiling of bone microstructure in a low calcium diet fed laying hens. Poultry Science 98 (10): 4359-4368. https://doi.org/10.3382/ps/pez271.

Johnson, C.H., J. Ivanisevic, and G. Siuzdak. 2016. Metabolomics: beyond biomarkers and towards mechanisms. Nature Reviews. Molecular Cell Biology 17 (7): 451-459. https://doi.org/10.1038/nrm.2016.25.

Khosla, S., and L.C. Hofbauer. 2017. Osteoporosis treatment: recent developments and ongoing challenges. The Lancet Diabetes and Endocrinology 5 (11): 898907. https://doi.org/10.1016/S2213-8587(17)30188-2. 
Kilpinen, L., F. Tigistu-Sahle, S. Oja, D. Greco, A. Parmar, P. Saavalainen, J. Nikkilä, M. Korhonen, P. Lehenkari, and R. Käkelä. 2013. Aging bone marrow mesenchymal stromal cells have altered membrane glycerophospholipid composition and functionality. Journal of Lipid Research 54 (3): 622-635. https://doi.org/10.1194/jlr.M030650.

Kim, W.K., S.A. Bloomfield, T. Sugiyama, and S.C. Ricke. 2012. Concepts and methods for understanding bone metabolism in laying hens. World's Poultry Science Journal 68 (1): 71-82. https://doi.org/10.1017/S0043933912000086.

Komori, T. 2010. Regulation of bone development and extracellular matrix protein genes by RUNX2. Cell and Tissue Research 339 (1): 189-195. https:// doi.org/10.1007/s00441-009-0832-8.

Li, C., Q. Li, Q. Mei, and T. Lu. 2015. Pharmacological effects and pharmacokinetic properties of icariin, the major bioactive component in Herba Epimedii. Life Sciences 126: 57-68. https://doi.org/10.1016/j.lfs.2015.01.006.

Lorentzon, M., and S.R. Cummings. 2015. Osteoporosis: the evolution of a diagnosis. Journal of Internal Medicine 277 (6): 650-661. https:/doi.org/10.1111/joim.12369.

Luo, D., J. Li, K. Chen, X. Rong, and J. Guo. 2018. Untargeted metabolomics reveals the protective effect of Fufang Zhenshu Tiaozhi (FTZ) on aginginduced osteoporosis in mice. Frontiers in Pharmacology 9: 1483. https://doi. org/10.3389/fphar.2018.01483.

Lv, H., F. Jiang, D. Guan, C. Lu, B. Guo, C. Chan, S. Peng, B. Liu, W. Guo, and H. Zhu. 2016. Metabolomics and its application in the development of discovering biomarkers for osteoporosis research. International Journal of Molecular Sciences 17 (12): 2018. https://doi.org/10.3390/ijms17122018.

Martin, T.J., and N.A. Sims. 2015. RANKL/OPG, critical role in bone physiology. Reviews in Endocrine \& Metabolic Disorders 16 (2): 131-139. https://doi.org/1 0.1007/s11154-014-9308-6.

Moayyeri, A., C. Cheung, K.C. Tan, J.A. Morris, A. Cerani, R.P. Mohney, J.B. Richards, C. Hammond, T.D. Spector, and C. Menni. 2018. Metabolomic pathways to osteoporosis in middle-aged women: a genome-metabolome-wide mendelian randomization study. Journal of Bone and Mineral Research 33 (4): 643-650. https://doi.org/10.1002/jbmr.3358.

Morris, H.A., R. Eastell, N.R. Jorgensen, E. Cavalier, S. Vasikaran, S. Chubb, J.A. Kanis, C. Cooper, and K. Makris. 2017. Clinical usefulness of bone turnover marker concentrations in osteoporosis. Clinica Chimica Acta 467: 34-41. https://doi. org/10.1016/j.cca.2016.06.036.

Nian, H., M. Ma, S. Nian, and L. Xu. 2009. Antiosteoporotic activity of icariin in ovariectomized rats. Phytomedicine 16 (4): 320-326. https://doi.org/10.1016/j. phymed.2008.12.006.

Patwardhan, V.G., A.V. Khadilkar, S.A. Chiplonkar, Z.M. Mughal, and V.V. Khadilkar. 2015. Varying relationship between 25-hydroxy-vitamin D, high density lipoprotein cholesterol, and serum 7-dehydrocholesterol reductase with sunlight exposure. Journal of Clinical Lipidology 9 (5): 652-657. https://doi. org/10.1016/j.jacl.2015.05.007.

Qi, S., J. He, H. Zheng, C. Chen, and S. Lan. 2019. Icariin prevents diabetesinduced bone loss in rats by reducing blood glucose and suppressing bone turnover. Molecules. 24 (10): 1871. https://doi.org/10.3390/molecules24101871.

Recker, R.R., J.M. Lappe, M. Davies, and D. Kimmel. 2018. Perimenopausal bone histomorphometry before and after menopause. Bone 108: 55-61. https://doi. org/10.1016/j.bone.2017.12.016.

Rodriguez-Navarro, A.B., H.M. McCormack, R.H. Fleming, P. Alvarez-Lloret, J. Romero-Pastor, N. Dominguez-Gasca, T. Prozorov, and I.C. Dunn. 2018. Influence of physical activity on tibial bone material properties in laying hens. Journal of Structural Biology 201 (1): 36-45. https://doi.org/10.1016/j. jsb.2017.10.011.

Saelao, P., Y. Wang, G. Chanthavixay, R.A. Gallardo, A. Wolc, J. Dekkers, S. Lamont, T. Kelly, and H. Zhou. 2019. Genetics and genomic regions affecting response to Newcastle disease virus infection under heat stress in layer chickens. Genes. 10 (1): 61. https://doi.org/10.3390/genes10010061.

Tarlton, J.F., L.J. Wilkins, M.J. Toscano, N.C. Avery, and L. Knott. 2013. Reduced bone breakage and increased bone strength in free range laying hens fed omega-3 polyunsaturated fatty acid supplemented diets. Bone. 52 (2): $578-$ 586. https://doi.org/10.1016/j.bone.2012.11.003.

Wang, Z., D. Wang, D. Yang, W. Zhen, J. Zhang, and S. Peng. 2018. The effect of icariin on bone metabolism and its potential clinical application. Osteoporosis International 29 (3): 535-544. https://doi.org/10.1007/s00198-017-4255-1.

Wei, Q., J. Zhang, G. Hong, Z. Chen, W. Deng, W. He, and M. Chen. 2016. Icariin promotes osteogenic differentiation of rat bone marrow stromal cells by activating the ERa-Wnt/ $\beta$-catenin signaling pathway. Biomedicine \& Pharmacotherapy 84: 931-939. https://doi.org/10.1016/j.biopha.2016.09.107.
Whitehead, C.C. 2004. Overview of bone biology in the egg-laying hen. Poultry Science 83 (2): 193-199. https://doi.org/10.1093/ps/83.2.193.

Whitehead, C.C., and R.H. Fleming. 2000. Osteoporosis in cage layers. Poultry Science 79 (7): 1033-1041. https://doi.org/10.1093/ps/79.7.1033.

Xu, J., M. Yao, J. Ye, G. Wang, J. Wang, X. Cui, and W. Mo. 2016. Bone mass improved effect of icariin for postmenopausal osteoporosis in ovariectomyinduced rats: A meta-analysis and systematic review. Menopause. 23 (10): 1152-1157. https://doi.org/10.1097/GME.0000000000000673.

Xu, Y., S. Chen, T. Yu, J. Qiao, and G. Sun. 2018. High-throughput metabolomics investigates anti-osteoporosis activity of oleanolic acid via regulating metabolic networks using ultra-performance liquid chromatography coupled with mass spectrometry. Phytomedicine. 51: 68-76. https://doi.org/10.1016/j. phymed.2018.09.235.

Zhang, G., L. Qin, W.Y. Hung, Y.Y. Shi, P.C. Leung, H.Y. Yeung, and K.S. Leung. 2006. Flavonoids derived from herbal Epimedium Brevicornum maxim prevent OVX-induced osteoporosis in rats independent of its enhancement in intestinal calcium absorption. Bone. 38 (6): 818-825. https://doi.org/10.101 6/j.bone.2005.11.019.

Zhang, M., Y. Wang, Q. Zhang, C. Wang, D. Zhang, J. Wan, and C. Yan. 2018. UPLC/Q-TOF-MS-based metabolomics study of the anti-osteoporosis effects of Achyranthes bidentata polysaccharides in ovariectomized rats. International Journal of Biological Macromolecules 112: 433-441. https://doi. org/10.1016/j.ijbiomac.2018.01.204.

Zhao, H., N. Zhao, P. Zheng, X. Xu, M. Liu, D. Luo, H. Xu, and D. Ju. 2018. Prevention and treatment of osteoporosis using Chinese medicinal plants: Special emphasis on mechanisms of immune modulation. Journal of Immunology Research 2018: 1-11. https://doi.org/10.1155/2018/6345857.

\section{Publisher's Note}

Springer Nature remains neutral with regard to jurisdictional claims in published maps and institutional affiliations.
Ready to submit your research? Choose BMC and benefit from:
- fast, convenient online submission
- thorough peer review by experienced researchers in your field
- rapid publication on acceptance
- support for research data, including large and complex data types
- gold Open Access which fosters wider collaboration and increased citations
- maximum visibility for your research: over $100 \mathrm{M}$ website views per year
At BMC, research is always in progress.
Learn more biomedcentral.com/submissions 